\title{
Literaturalisierung der Religion?
}

\author{
Anmerkungen zu Martin Walsers Über Rechtfertigung, eine \\ Versuchung
}

Matthias Neugebauer

Mit seinem Büchlein Über Rechtfertigung, eine Versuchung ist Martin Walser etwas gelungen, was im weitesten Sinne theologischen Texten in den letzten Jahre kaum mehr gelingt: im Feuilleton, in Fernseh- und in Radiosendungen wurde der Band ausführlich besprochen und Walsers Standpunkt diskutiert, es gab Seminare und es erschien sogar ein eigener Band mit theologischen Erwiderungen. Insbesondere aus der Zunft der Theologen gab es bei aller obligaten Kritik anerkennende und insgesamt wohlwollende Rückmeldungen, die dem Nicht-Theologen Walser u.a. bescheinigten, einen der aufregendsten theologischen Texte der letzten Jahre verfasst zu haben.

Man kann sich fast des Eindrucks nicht erwehren, dass gerade unter Theologen eine Stimmung der Dankbarkeit sich breitmachte, eine Dankbarkeit, die zeigt, welch' grosser Durst nach medialer Aufmerksamkeit in den theologischen Fachkreisen herrscht, die oft Binnendiskussionen führen; bzw. umgekehrt, wie drastisch ein gefühlter oder wirklicher Bedeutungsverlust das Selbstverständnis einer Zunft geprägt hat, wenn sie sich vor captationes fast überschlägt, findet eines ihrer - zugegeben zentralen -, Stichworte den Weg an die Oberfläche einer breiteren Wahrnehmung.

Aber hat Walsers Text der Theologie tatsächlich einen Gefallen getan? Jedenfalls wirft Walsers Band - und das will er auch - eine ganze Reihe von Fragen auf. An dieser Stelle soll das Augenmerk kurz auf drei Punkte gelenkt werden: Erstens Walsers offenkundige Sympathie für die Dialektische Theologie und seine ebenso deutliche Kritik an der Liberalen Theologie, zweitens das unverkrampfte Zusammenstellen und -lesen der PFARRERSÖHNE Karl Barth und Friedrich Nietzsche und schliesslich sein Vorschlag, religiöse Texte, v.a. auch die Bibel, als Literatur zu lesen. 
I.

Gegen einen in weiten Kreisen zur Gewohnheit gewordenen Atheismus erinnert Walser an den Mangel, welcher derjenige verspürt, der Gott zwar nicht mehr hat, aber noch darum weiss, was einen Gott zu haben, bedeuten würde. Die gesellschaftlichen Folgen dieses Mangels erklären sich für Walser aus der engen Verbindung des Gottesgedankens mit der Idee der Rechfertigung: Fehlt die Rechtfertigung von oben, so kommt es zu einem gravierenden Rechtfertigungsmangel, der umschlägt in eine mehr oder weniger aggressive Kultur des Rechthabens und der Rechthaberei, für die im Übrigen auch Nietzsche angeführt wird, der nicht nur für heute und morgen, sondern nach eigenen Angaben für tausende Jahre Recht behalten wollte.

Die Kultur des Rechthabens, des Rechthabenmüssens - und hier wird der Kulturprotestantismus das erste Mal indirekt angezählt -, hat seinen Bezugspunkt im Selbst des Menschen, in dem, was er leistet, erarbeitet, besitzt, kann und tut. Das Forum der Rechtfertigung ist nicht mehr ein theologisches, sondern ein zutiefst menschliches, das nunmehr in den Niederungen der talkshows (statt der Beichte) gelandet ist. Der aggressiven Kultur des Rechthabens entgegen stellt Walser die Idee der Selbstwiderlegung, dass also alles, was behauptet wird, einen strengen Gegentest der Selbstwiderlegung zu durchlaufen hätte. Konkret meinte das, dass - wie z.B. das "sed contra" in der Scholastik - in den Prozess der Behauptung immer auch das Gegenteil dessen, was behauptet wird, einer vorurteilsfreien Prüfung unterzogen werden sollte, mithin eben der Versuch einer rationalen Selbstwiderlegung.

Walsers kritischer Kulturdeutung wird man kaum widersprechen wollen. Insbesondere der Befund, dass zwischen dem gesellschaftlich verbreiteten Rechthabenmüssen und dem Verlust an Rechtfertigung von oben ein Zusammenhang besteht, ist in theologischer Perspektive durchaus bedenkenswert. Welcher Theologe möchte widersprechen, wenn ein Schriftsteller verkündigt, dass es das Verdienst der Religion sei, die Frage, ob wir uns durch unsere Werke oder unseren Glauben rechtfertigen, wachgehalten zu haben. Wenn er dabei, als die grossen Wachhalter der Rechtfertigungsidee, Paulus, Augustinus, Luther und Karl Barth aufzählt, erwächst in mir - als einem Theologen, der auch die Tradition der Liberalen Theologie zu schätzen weiss - aber Widerspruch. Insbesondere Karl Barth wird nämlich durch den ganzen Text hindurch nachgerade zur Ikone der wahren Theologie stilisiert, die nicht den Versuchungen der Zeitgeistopportunität erlegen sei, 
sondern dem es gelungen sei, aus dem Zirkel des Rechthabenmüssens auszusteigen.

An dieser Stelle scheinen bei allem Respekt gegenüber Walsers Reflexionen über die Rechtfertigung und seinem Ringen um Gott gewisse Einseitigkeiten durch den Text.Verbleibt der Katholizismus fast unsichtbar im Hintergrund, so werden am Orte des Protestantismus die Fronten klar markiert und besetzt. Dialektische Theologie à la Karl Barths Römerbrief: gut; Liberale Theologie à la Schleiermacher, von Harnack, Troeltsch und Weber: böse resp. Religion ssoft‘, weil es v.a. Schleiermacher gewesen sei, der eine dem Religiösen nicht zuträgliche, ja schädliche Entspannung und Verwässerung desselben zu verantworten habe.

Das sind m.E. überaus starke und einseitige Entgegensetzungen. Tritt einem, wenn man Schleiermachers Predigten, Monologe oder Reden liest, tatsächlich nur eine 'Religion soft « entgegen? Und kann, wer sich auf die gedankliche Strenge und Belesenheit eines Ritschl, die theologische Prosa von von Harnacks dogmengeschichtlichen Auseinandersetzungen, Emmanuel Hirschs treffende theologische Formulierungen und Tillichs Interpretation der Rechtfertigungslehre ("annehmen, dass wir angenommen sind ") gründlich einlässt, die Liberale Theologie wirklich nur als Verlustgeschichte beschreiben?

Die Apotheose Karl Barths wird dadurch noch fragwürdiger, dass nur ganz bestimmte Züge von dessen Denken in den Vordergrund gerückt werden. Der späte Barth interessiert im Grunde nicht, genauso wie bei Paulus, Augustin und Calvin für Walser v.a. die Erwählungs- resp. nur die Prädestinationslehre von Interesse zu sein scheint. Der grosse Wachhalter Luther wird insgesamt recht stiefmütterlich behandelt, aber es steht zu vermuten, dass der Deus absconditus bei Walser wiederum auf mehr Interesse stiesse als der Deus revelatus.

$\mathrm{Ob}$ mit Blick auf Barths Dialektik, Kierkegaards Paradox oder Hölderlins Suchen - immer sind Walsers Auseinandersetzungen und Zusammenstellungen von einem seltsamen faible für das Opake, das schwer zu Fassende, das Entzogene, Undurchsichtige, Unfassbare und Unbegreifliche bestimmt.

So bleibt man am Schluss etwas ratlos zurück mit der Frage:Von was für einer Religion ist da eigentlich die Rede? Von der christlichen? Das mag die Kompilation der behandelten Autoren nahe legen. Doch Jesus Christus kommt mit keinem Wort zu Wort oder vor, dafür allerdings ein prominenter Kritiker des Christentums: Friedrich Nietzsche nämlich, der wie kein anderer vom Tode Gottes gesprochen sowie das Christentum leidenschaftlich bekämpft hat. Das führt auf den zweiten Punkt. 
II.

Mit Martin Walsers Über Rechtfertigung, eine Versuchung ist sie also (wieder einmal) im Feuilleton angekommen: Nietzsches mächtige Prophezei vom Tode Gottes; und dessen Folgen. Jetzt ist es Martin Walser, der als Trittbrettfahrer Nietzsches auftritt und die >Gottist-tot-These Gleichzeitig outet sich Walser - wie gesehen -, als Karl-Barth-Fan, besser gesagt, als Begeisterter des frühen Barth und dessen Römerbrief, der 1919 in der ersten und 1922 in der zweiten Auflage erschien. Tertia comparationis sind ihm zum einen Barths Rede von der Entzogenheit Gottes, die Hohlraum-Metaphorik und das unbekannte und ewige Futurum Gottes, das furr Walser durchaus parallelisierbar ist mit der Nachricht vom Tode Gottes, die Nietzsche Zarathustra in den Mund gelegt hat. Jedenfalls stellt Walser durchaus in Frage, ob denn Nietzsches Prophezei vom Tode Gottes tatsächlich so verschieden sei von Barths theologischer Rede, die Gott immer wieder als den Unbekannten und Entzogenen, als die unmögliche Möglichkeit aufruft. Zum anderen glaubt Walser Nietzsche und Barth miteinander ins Gespräch bringen zu müssen, weil - und diese Tatsache vermag Walser offenbar über die Massen zu faszinieren - sowohl Karl Barth als auch Friedrich Nietzsche Pfarrersöhne sind.

Beides wirkt auf den ersten Blick originell und provokant. Das lädt zu näherem Hinsehen ein. Denn schon der erste Punkt ist alles andere als selbstverständlich: Nietzsches >Gott-ist-tot-These und Barths >Totaliter-aliter-Votum stehen auf zwei ganz unterschiedlichen Sockeln. Nietzsches und Barths Suchbewegungen ruhen auf jeweils ganz verschiedenen Fundamenten und verfolgen ganz unterschiedliche Interessen. Barth kommt aus der Tradition der Liberalen Theologie, von der er sich dann leidenschaftlich absetzt. Er hat es sich auf die Fahnen geschrieben, mit Verwegenheit den verbürgerlichten Zustand seiner Kirche zu entlarven und die Energien des Evangeliums wieder zu erwecken durch Absage an begriffliche Konzepte und die Beschwörung einer unmöglichen, unsichtbaren und unbekannten Kirche (die Kirche Jakobs), an die nur ohne Hoffnung auf Hoffnung hin geglaubt werden kann. Nietzsche hingegen kommt aus der Altphilologie und -gräzistik; und gilt auch dort als Aussenseiter. Einer der führenden Fachkollegen seiner Zeit - Ulrich von Wilamowitz-Moellendorf - hat Nietzsches Die Geburt der Tragödie aus dem Geiste der Musik von 1872 seinerzeit gründlich verrissen. Von den Theologischen Diskursen seiner Zeit hat Nietzsche nur entfernt eine Ahnung. Er kennt hier meist nur Schlagwörter. Weder 
Schleiermacher, Baur, Biedermann, Daub, Pfleiderer noch Ritschl hat er je gründlich gelesen. $\mathrm{Zu}$ den wenigen Theologen seiner Zeit, mit denen er sich näher auseinandergesetzt hat, gehören David Friedrich Strauss und Franz Overbeck. Mit dem ersten hat er gnadenlos in seiner Ersten Unzeitgemässen Betrachtung abgerechnet; mit dem zweiten - der sich öffentlich zum Unglauben bekannte - war er eng befreundet. Ansonsten wird pauschal, mit Gefährlichkeit und grossem Stil, kurz: mit dem Hammer, philosophiert resp. polemisiert.

Der Gott, der Nietzsche interessiert, ist nicht der christliche Gott. Nietzsches erstes und letztes Interesse gilt einer Dionysischen Weltanschauung (so der Titel einer erst postum veröffentlichen Schrift aus dem Jahr 1870; die Dionysos-Dithyramben, die Nietzsche 1888 zur Publikation vorbereitete, stehen ganz am Ende seines Werkes). Die Opakheit des unbekannten und unerkennbaren Gottes Barths und Nietzsches Ur-Eines und Dionysisches (sein in modo aeternitatis gedachter Wille zur Macht) sind grundverschiedene Figuren. Und wenn Walser im Spätwerk bei Nietzsche eine Gottessehnsucht im Sinne Augustins bis Barth vindiziert, so darf keinesfalls übersehen werden, dass Nietzsche sich hier selbst als die Erfüllung dieser Sehnsucht in Szene setzt: »Ich bin deine Wahrheit ...» ist bekanntlich der letzte Satz der Dionysos-Dithyramben, mit denen Nietzsche sein imaginäres Zwiegespräch mit Zarathustra beendet, als Dionysos, als der Dionysos, der gegen den Gekreuzigten gekämpft hat, wie Ecce homo endet.

So bleibt es dabei, dass Barth und Nietzsche am ehesten in der Form, keinesfalls in der Sache konvergieren. Und im Blick auf die Form fallen in der Tat Übereinstimmungen ins Auge:Widerstand gegen Systematisierung, begriffliche Internierung und argumentative Domestizierung des Ewigen; stattdessen eine fast expressionistische und experimentelle Sprache Barths im Römerbrief und kunstvolle wie offene Aphorismen und Dithyramben bei Nietzsche. Beider Sprache ist mehr Dichtung denn Argumentation, was Walser mit der durchaus gelungenen Formel Tanzschritte der Dialektik einfängt. Das darf aber nicht darüber hinwegtäuschen, dass der Gott, dem Karl Barth entgegentanzt, ein anderer ist, als Nietzsches Gott.

Zweitens darf an dieser Stelle nicht vergessen gehen, dass auch das Vergleichsmoment PFARRERSÖHNE (Walser schlägt dies als Titel eines von ihm vorgestellten Barth-Nietzsche-Seminars vor) mit Vorsicht zu geniessen ist. Tatsächlich starb Nietzsches Vater bereits 1849, da war Nietzsche noch nicht einmal fünf Jahre alt. Aufgewachsen ist er dann in Naumburg bei seiner Mutter und Schwester, mit seiner Grossmutter und zwei unverheirateten Tanten; einen grösseren Kon- 
trast zu einem Pfarrhaushalt, wie der, in dem Karl Barth aufwuchs, kann man sich fast nicht vorstellen. Entsprechend unausgeprägt war Nietzsches Entschluss, Theologie zu studieren; bekanntlich hat er bereits nach dem ersten Semester abgebrochen. DerVergleichspunkt Pfarrersöhne ist also wenig überzeugend. Dies umso mehr, als ja auch liberale Theologen wie Adolf von Harnack, Albrecht Ritschl, Friedrich Schleiermacher oder Paul Tillich (der für Walser an anderer Stelle auch schon mal unter der Rubrik »abschreckend « firmieren kann) Pfarrersöhne waren.

Was also fasziniert Walser? Das Religiöse oder das mit dem Religiösen aufgeworfene Problem der Versprachlichung seiner selbst? Damit kommen wir zum Dritten und Letzten.

III.

$\mathrm{Zu}$ reden gegeben hat, dass Walser die Bibel resp. religiöse Texte (gross unterschieden wird da nicht) als Literatur liest. Was heisst das genau? Freilich gibt es in der Bibel und auch in den auf sie bezogenen theologischen Texten grosse Unterschiede, was das sprachliche Niveau der Autoren anbelangt. Und mit Recht werden das Buch Hiob, der Psalter und der Lukanische Bericht der Geburt Jesu als literarische Höhepunkte aufgerufen. Und keiner wird je behaupten, dass es sich bspw. bei 1. Chronik, Kap. 1-12 um grosse Literatur handelt.

Allerdings erheben sich auch hier Fragen. Wenn Walser meint, dass Religion eine Ausdrucksart sei, wie Literatur, Musik und Malerei, wirkt das in dem Moment problematisch, wenn in Rechnung gestellt wird, dass sich die Religion eben genau dieser Ausdrucksarten bedient, eben der Literatur, der (Ton-)Kunst und der Malerei. Das Verhältnis von Religion und Literatur, Kunst und Malerei ist ein anderes als das von Literatur, der Kunst und der Malerei untereinander.

Hinzu kommt ein Zweites: Kann man trennen zwischen hoher Poesie, Ausdruckskraft sowie gelungener Formulierung und dem Geist, der alle diese Ambitionen regt und regiert, ohne in die Figur einer ars gratia artis zu geraten? Luther hat gesagt, es sei entscheidend, bei der Bibellektüre auf das zu achten, "was Christum treibet«. Das ist kein literarischer, sondern ein zutiefst religiös-hermeneutischer Schlüssel, der in keiner Weise nur auf das Literarische abstellt. Und "was Christum treibet « kann in den vollkommenen Formulierungen des Psalters aufgefunden werden (den Luther bekanntlich konse- 
quent christologisch interpretiert) wie im z.T. hölzernen Ringen der Theologen der Briefe.

Wenn Walser vorschlägt, die Bibel oder religiöse Autoren zu lesen wie Dostojewski oder Flaubert, so kommt mir das ein wenig vor wie Lenin, der Hegel liest. Im Polit-Unterricht der DDR mussten wir als Schüler ein Buch von Lenin lesen, in dem u.a. Konspekte seiner Hegel-Lektüre abgedruckt waren. Und dort hiess es sinngemäss, dass er (also Lenin) sich bemühe, Hegel im Allgemeinen materialistisch zu lesen, was bedeute, dass er den lieben Gott, das Absolute etc. grösstenteils weglasse. Wir wissen, was dabei heraus gekommen ist.

Keineswegs soll aus Walser ein Materialist oder Leninist gemacht werden. Und doch erinnert mich die Ent-Heiligung und Literaturalisierung Gottes durch den Literaten Walser an die Ent-Idealisierung des Idealisten Hegel durch den Materialisten Lenin. Einer solchen Literaturalisierung Gottes ist m.E. mit grosser theologischerVorsicht zu begegnen. Denn schafft Walser mit seiner Literatur-These nicht an dem mit, was er den (liberalen) Theologen vorwirft, nämlich, dass sie die Auf- und Angeregtheit durch das, was in der christlichen Theologie immer mit dem Namen, Titel, Symbol etc. \esus Christus verbunden ist, in die Lebens-Welt holen und das Moment des Unverfügbaren begrifflich etc. einfrieren wollen; nur dass Walser dabei keine Begriffe und keinen Heiland kennt, sondern eben nur Literatur?

So bleibt zum Schluss weniger Euphorie und Dankbarkeit darüber, dass ein prominenter Literat sich des Themas Religion und Rechtfertigung angenommen hat, sondern eher ein staunendes Nachdenken und - trotz aller Einreden - eine geheime Faszination dafür, wie theologisches Suchen auch aussehen kann.

— Dr. Matthias Neugebauer ist Privatdozent an der Universität Zürich und Pfarrer in der Reformierten Kirchgemeinde Sursee. 